\title{
Metastatic renal cell carcinoma mimicking a schwannoma in a dorsal root ganglion: case report
}

\author{
Jason K. Wasserman, MD, PhD,, Eve C. Tsai, MD, PhD, FRCSC, ${ }^{1}$ Rafael Glikstein, MD, ${ }^{3}$ \\ Kien T. Mai, MD, ${ }^{1}$ and Gerard H. Jansen, MD ${ }^{1}$ \\ 1Division of Anatomical Pathology, Pathology and Laboratory Medicine, University of Ottawa; and 2Division of Neurosurgery and \\ ${ }^{3}$ Department of Diagnostic Imaging, The Ottawa Hospital, Ottawa, Ontario, Canada
}

\begin{abstract}
Peripheral nerve tumors are soft-tissue tumors that can occur in any nerve throughout the body. The majority of peripheral nerve tumors arise from elements of the nerve sheath with the two most common being neurofibromas and schwannomas. More than $90 \%$ of all peripheral nerve tumors are benign. When there is peripheral nerve involvement in metastatic carcinoma, it is often via contiguous spread from the primary mass; hematogenous seeding to a peripheral nerve is seldom seen. In this report the authors describe the even rarer case of metastatic renal cell carcinoma mimicking a schwannoma in a dorsal root ganglion. Cases from the literature show the rarity of this finding and its late clinical appearance. Given that survival in patients with metastatic carcinoma continues to increase, dorsal root ganglion metastasis may become more common over time.
\end{abstract}

http://thejns.org/doi/abs/10.3171/2014.9.SPINE131075

KEY WORDS renal cell carcinoma; metastasis; dorsal root ganglion; schwannoma; dumbbell lesion; oncology

$\mathrm{P}$ ERIPHERAL nerve tumors are soft-tissue tumors that can occur in any nerve throughout the body. The majority of peripheral nerve tumors arise from elements of the nerve sheath with the two most common being neurofibromas and schwannomas. ${ }^{6}$ More than $90 \%$ of all peripheral nerve tumors are benign. ${ }^{4}$ When there is peripheral nerve involvement in metastatic carcinoma, it is often via contiguous spread from the primary mass; hematogenous seeding to a peripheral nerve is seldom seen. In this report we describe the even rarer case of metastatic renal cell carcinoma (RCC) mimicking a schwannoma in a dorsal root ganglion (DRG) and review the literature describing other cases of metastatic disease involving the DRG.

\section{Case Report}

History and Examination

During routine follow-up CT scanning of the thorax, a 67-year-old woman with known extensively metastasized RCC was found to have a solitary mass involving the T-5 DRG 6.5 years after discovery of her primary tumor
(Fig. 1A). A retrospective analysis of a CT scan obtained 2 years earlier identified the same lesion and showed that it had increased in size during the interval (Fig. 1B). MRI of the spine confirmed a $16 \times 8-\mathrm{mm}$ dumbbell-shaped mass in the right T5-6 intervertebral foramen. The mass displayed homogeneous low T1 signal (Fig. 1C) and high T2 signal (Fig. 1D) with avid homogeneous enhancement (Fig. 1E). There was smooth remodeling and expansion of the intervertebral foramen, but the surrounding bones were not involved. The mass extended into the right lateral epidural space, effacing the epidural fat and thecal sac, and minimally toward the cord, which was mildly deviated but not compressed. On sagittal T1- and T2-weighted imaging, the mass had a hypointense core (Fig. $1 \mathrm{~F}$, arrow). The T2 signal of the foraminal lesion was noticeably higher than the known renal metastases in the contralateral kidney (not shown). Radiologically the tumor was most consistent with a schwannoma.

The patient was subsequently referred to a neurosurgeon. The patient denied any weakness, sensory changes, or bladder or bowel dysfunction. The neurological examination findings were unremarkable, and no periph- 

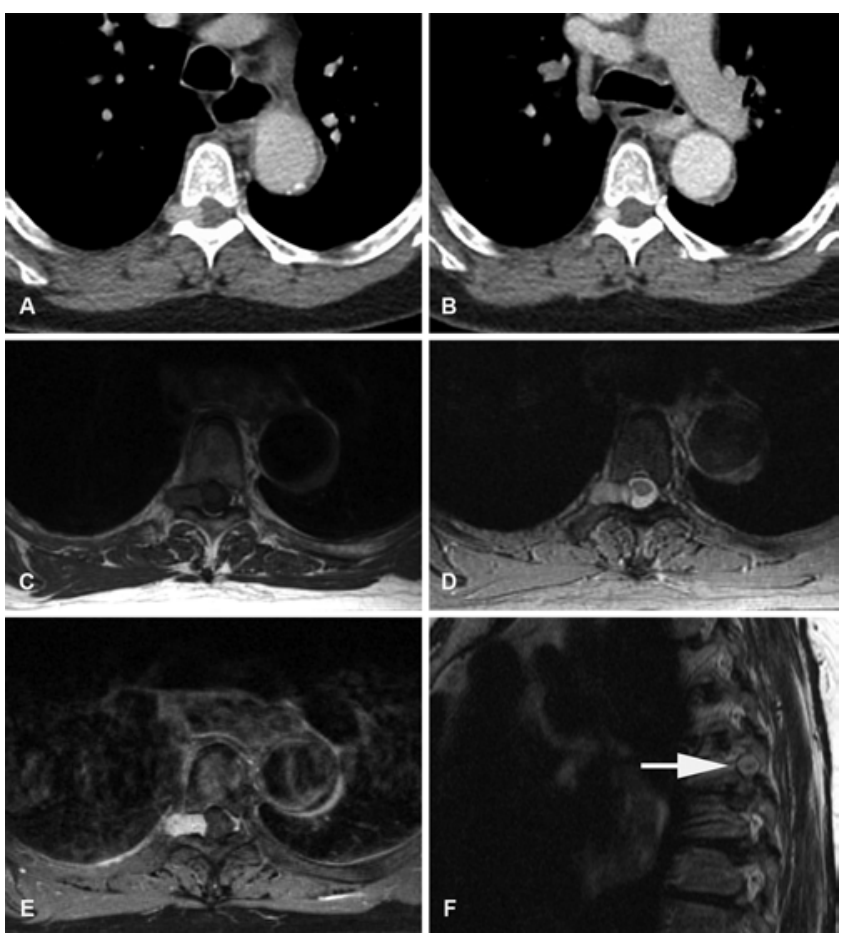

FIG. 1. A and B: CT scans showing a solitary lesion involving the T-5 DRG $(A)$ and the same lesion 2 years prior (B). C-E: Axial T1-weighted MR image (C), T2-weighted image (D), and image obtained after gadolinium administration (E). F: Sagittal T1-weighted image showing the hypointense core (arrow).

eral nerve or spinal cord symptoms were present; motor strength was $5 / 5$ throughout, reflexes were normal and symmetrical, toes were downgoing bilaterally, sensation was intact, and gait was normal.

\section{Operation}

Given that the lesion was growing, the decision was made to proceed with surgical biopsy for diagnosis. Intraoperatively, the proximal nerve was visualized exiting the foramen, and there did not appear to be a tissue plane between the tumor mass and the nerve. Distal to the tumor mass the nerve appeared to branch into multiple fascicles; the resection margin was distal to these fascicles. The tumor specimen was sent for pathological analysis. Intraoperative blood loss was $800 \mathrm{ml}$, with most of the blood loss coming from epidural veins; the tumor did not bleed significantly.

\section{Pathological Findings}

Microscopy of the tumor revealed a mass with an attached spinal nerve, encapsulated by part of the normal DRG structures (Fig. 2A). While normal DRG cells were found at the periphery (Fig. 2C, arrow), the remainder of the ganglion had been replaced by large fields of tumor cells with optically empty cytoplasm and round to oval, fairly regular, nuclei. Many thin-walled capillaries were also visualized within the tumor. The tumor extended to the proximal surgical margin.

Immunohistochemical analysis showed that both the tumor and the adjacent nerve were positive for the mesen-
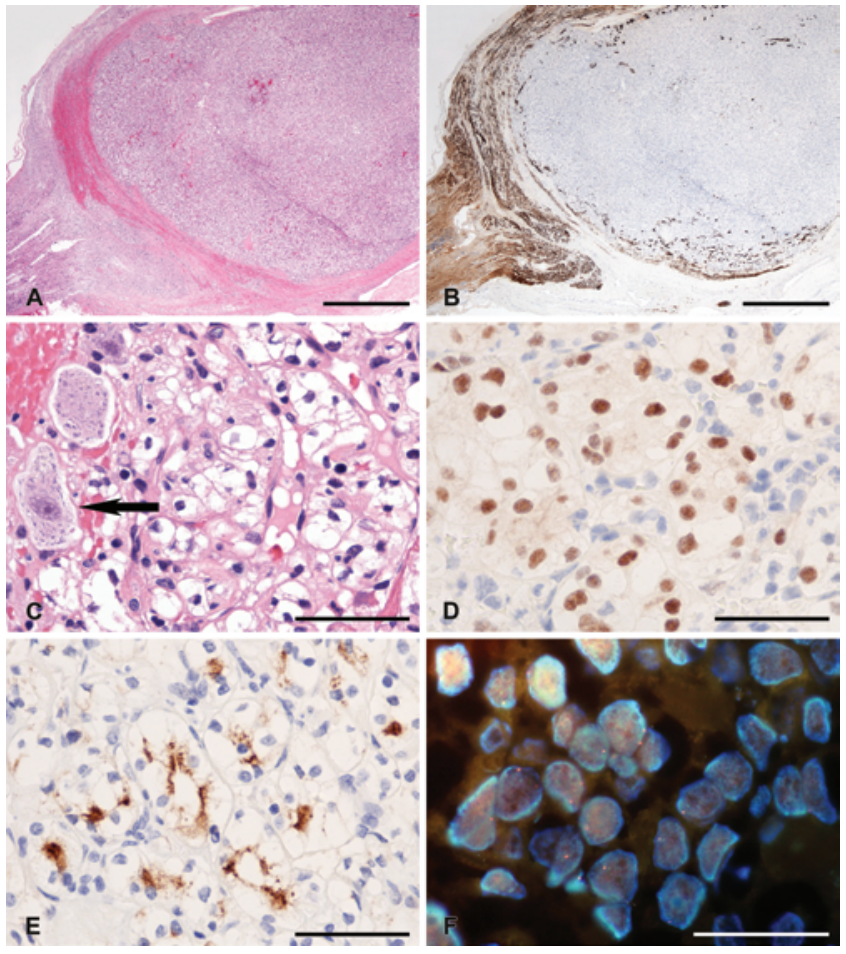

FIG. 2. Metastatic renal cell carcinoma infiltrating a dorsal root ganglion. A: Low-power overview. H \& E. B: Only the adjacent nerve is positive for the Schwann cell maker S100. C: High-power view with preexisting neurons at margin of tumor (arrow). H\& E. D: Tumor cells positive for PAX-8. E: Tumor cells positive for RCC. F: FISH analysis showing loss of the short arm of chromosome 3 in the tumor cells. Bar = $1000 \mu \mathrm{m}(\mathrm{A}$ and $\mathrm{B}) ; 50 \mu \mathrm{m}(\mathrm{C}-\mathrm{E}) ; 25 \mu \mathrm{m}(\mathrm{F})$.

chymal marker vimentin (not shown), while only the adjacent nerve was positive for the Schwann cell marker S100 (Fig. 2B). The tumor cells were positive for PAX-8 (Fig. 2D), the RCC marker (Fig. 2E), and CD10 (not shown). Because most cases of RCC are associated with a deletion of the von Hippel Lindau gene on the short arm of chromosome 3, fluorescence in situ hybridization (FISH) was performed which confirmed the loss of the $3 \mathrm{p} 25$ region in all tumor cells. Taken together, these findings are consistent with an RCC metastasis.

At the 3-month follow-up, the patient was free of motor, sensory, or autonomic spinal cord or peripheral nerve symptoms. Neither radiotherapy nor chemotherapy was given at this point. Further resection was offered to the patient; however, because she had other metastatic lesions that were progressing and because she was asymptomatic with respect to the thoracic lesion, the patient refused further treatment. Five months postsurgery the patient was started on sunitinib malate to control growth of metastatic disease elsewhere. Currently, 1 year after surgery, there is no recurrence of the DRG lesion, and the patient has no peripheral nerve or spinal cord symptoms.

\section{Discussion}

Most tumors involving the spinal nerve roots and DRG are primary neoplasms that arise from components of the neural sheath, schwannomas being the most common. ${ }^{7}$ 
Schwannomas arise from neoplastic Schwann cells that proliferate and displace the normal components of the nerve to the periphery. Normally, Schwann cells are responsible for myelinating axons in peripheral nerves and are analogous to oligodendrocytes in the central nervous system. Neurofibromas are another common type of primary peripheral nerve tumor, which arise from the proliferation of several cell types normally present in the neural sheath: Schwann cells, perineuronal cells, and fibroblasts.

The central nervous system is frequently affected by metastatic tumor. The peripheral nervous system is also often affected by tumor elsewhere, by perineural and neural growth directly in or around a tumor-factors that are often used in grading tumors and are considered a poor prognostic sign. Hematogenous spread to peripheral nerves is rare, and despite the frequency of metastatic cancer in general, metastatic cancer to the DRG is even rarer. To date, only 7 reports of a total of 9 cases have been reported in the literature (Table 1). The first part of this series comprises 3 autopsy reports documenting 1654 autopsies, in which a total of 5 cases of DRG metastasis were found. ${ }^{1,3,13}$ For those studies with details, the lesions were asymptomatic, and the average interval between primary tumor diagnosis and autopsy was less than a year. These findings are likely biased by the fact that these studies were based on autopsies. The study by Van Ketel is the most extensive per case, as serial sections of 4 DRGs per autopsy were obtained..$^{13}$ As not all DRGs in all studies were examined, it is certainly possible that subclinical/ asymptomatic metastasis to a DRG occurs more frequently than only 5 of 1654 cases.

The 4 other studies, which are case reports, as well as our case, had some clinical manifestation. Two of these 5 cases were due to a primary adenocarcinoma ${ }^{10,14}$ and 2 were due to an RCC (including our case). ${ }^{11}$ Clinical details of presentation were available for 4 of the 5 patients. Three of these 4 patients presented with radicular pain. On average the 4 patients with clinical data presented 6 years and 4 months after the diagnosis of the primary tumor.

Combining the clinical and autopsy evidence, it seems that clinically significant metastasis to the DRG is a late and rare occurrence and that metastases are likely very slow growing at this location. The reason for this is unclear. One possibility is that metastases to the DRG go unnoticed because they are small, they do not cause symp- toms, or the symptoms such as radicular pain are attributed to other aspects of the disease, and that therefore the true frequency of DRG metastases is higher.

Management in our case was guided initially by radiological findings that led to a presumptive diagnosis of schwannoma. Indeed, many of the radiological features of the tumor identified in our patient were characteristic for an extramedullary intradural schwannoma. Specifically, the extra- and intraforaminal location and dumbbell shape of the tumor were consistent with a slow-growing, benign mass that extended through the intervertebral foramen with some associated remodeling. ${ }^{7}$ Moreover, the tumor in our case was consistent with a small schwannoma in that it showed homogeneous enhancement after gadolidium. ${ }^{8}$ However, the central hypointensity on sagittal T1weighted images differed from the previously described schwannoma "target sign" and may have represented a flow void caused by the highly vascular tumor. ${ }^{5}$ Conversely, the tumor was also consistent with RCC, which tends to be hypo- to isointense on T1-weighted images, iso- to hyperintense on T2-weighted images, and avidly enhancing. ${ }^{9}$ In hindsight, the radiological features in our case corresponded well with the histological appearance of the tumor: a vascular neoplasm, centrally infiltrating the DRG, and displacing the normal parenchyma to the periphery.

For most patients with spinal nerve tumors, surgery is indicated for symptom relief. Patients often present with symptoms of a radiculopathy, including weakness, paresthesia, and pain. ${ }^{2}$ Even though she was asymptomatic, our patient was treated surgically to obtain a diagnosis because the tumor was increasing in size and was starting to compress the spinal cord. The procedure was uneventful. Subsequent pathological analysis of permanent sections, however, revealed a metastatic lesion with positive margins.

Given the ever-increasing survival in cancer patients and the fact that DRGs are sites that, from a clinical point of view, are involved late in metastasis, it may well be that the frequency of DRG metastasis will increase in the future. This report therefore highlights the need to always include metastasis in the differential diagnosis for a new lesion in a patient with a known primary cancer, even when the lesion has the typical presentation of another common disease, or if survival after initial disease is long.

TABLE 1. Review of cases in the literature

\begin{tabular}{llllcl}
\hline \multicolumn{1}{c}{ Authors \& Year } & \multicolumn{1}{c}{ Study } & Primary Organ & \multicolumn{1}{c}{ Primary Tumor } & Interval (yrs) $^{*}$ & Symptoms $^{2}$ \\
\hline Chason et al., 1963 & 1096 autopsy cases & Unknown & Unknown & Unknown & Unknown \\
\hline Van Ketel, 1979 & 58 routine autopsy cases & Uterus & Cervical carcinoma & 1 & Unknown \\
\hline Johnson, 1977 & 500 routine autopsies & Lung & Small cell & 0.25 & No \\
\hline Wigfield et al., 2003 & Case report & Colon & Adenocarcinoma & 1 & No \\
\hline Uchida et al., 2008 & Case report & Colon & Adenocarcinoma & 12 & Yes \\
\hline Schultz et al., 2009 & Case report & Uterus & Adenocarcinoma & 4 & Yes \\
\hline Shakeel et al., 2009 & Case report & Breast & Ductal carcinoma & 1.5 & Yes \\
\hline Current case & Case report & Kidney & RCC & 3 & Yes \\
\hline
\end{tabular}

* In patients without symptoms, interval refers to the time between primary tumor diagnosis and autopsy or surgery. In patients with symptoms, interval refers to the time between primary diagnosis and symptom onset. 


\section{References}

1. Chason JL, Walker FB, Landers JW: Metastatic carcinoma in the central nervous system and dorsal root ganglia. A prospective autopsy study. Cancer 16:781-787, 1963

2. Jinnai T, Koyama T: Clinical characteristics of spinal nerve sheath tumors: analysis of 149 cases. Neurosurgery 56:510 515, 2005

3. Johnson PC: Hematogenous metastases of carcinoma to dorsal root ganglia. Acta Neuropathol 38:171-172, 1977

4. Kim DH, Murovic JA, Tiel RL, Kline DG: Operative outcomes of 546 Louisiana State University Health Sciences Center peripheral nerve tumors. Neurosurg Clin N Am 15:177-192, 2004

5. Koga H, Matsumoto S, Manabe J, Tanizawa T, Kawaguchi N: Definition of the target sign and its use for the diagnosis of schwannomas. Clin Orthop Relat Res 464:224-229, 2007

6. Louis DN, Ohgaki H, Wiestler OD, Cavenee WK, Burger PC, Jouvet A, et al: The 2007 WHO classification of tumours of the central nervous system. Acta Neuropathol 114:97-109, 2007

7. Ozawa H, Kokubun S, Aizawa T, Hoshikawa T, Kawahara C: Spinal dumbbell tumors: an analysis of a series of 118 cases. J Neurosurg Spine 7:587-593, 2007

8. Pilavaki M, Chourmouzi D, Kiziridou A, Skordalaki A, Zarampoukas T, Drevelengas A: Imaging of peripheral nerve sheath tumors with pathologic correlation: pictorial review. Eur J Radiol 52:229-239, 2004

9. Prasad SR, Humphrey PA, Catena JR, Narra VR, Srigley JR, Cortez AD, et al: Common and uncommon histologic subtypes of renal cell carcinoma: imaging spectrum with pathologic correlation. Radiographics 26:1795-1810, 2006
10. Schulz M, Lamont D, Muthu T, Hussain Z, Balakrishnan $\mathrm{V}$ : Metastasis of breast cancer to a lumbar spinal nerve root ganglion. Spine (Phila Pa 1976) 34:E735-E739, 2009

11. Shakeel M, Kumaravel M, Mackenzie JM, Knight DJ: An uncommon cause of sciatica. J Coll Physicians Surg Pak 19:127-129, 2009

12. Uchida K, Kobayashi S, Yayama T, Muramatsu J, Kurokawa T, Imamura Y, et al: Metastatic involvement of sacral roots from uterine carcinoma: a case report. Spine J 8:849-852, 2008

13. Van Ketel BA: De Menselijke Spinale Ganglia: Een Pathologisch-Anatomisch Onderzoek [thesis]. Utrecht, the Netherlands: Elinkwijk Press, 1979

14. Wigfield CC, Hilton DA, Coleman MG, Whitfield PC: Metastatic adenocarcinoma masquerading as a solitary nerve sheath tumour. Br J Neurosurg 17:459-461, 2003

\section{Author Contributions}

Conception and design: Jansen, Wasserman. Acquisition of data: Wasserman, Tsai, Glikstein, Mai. Analysis and interpretation of data: Jansen, Wasserman, Tsai, Glikstein. Drafting the article: Jansen, Wasserman, Tsai, Glikstein. Reviewed submitted version of manuscript: Wasserman, Tsai, Glikstein, Mai. Study supervision: Jansen.

\section{Correspondence}

Gerard H. Jansen, Eastern Ontario Regional Laboratory, Civic Campus - Lab Med Building Rm. 121, 1053 Carling Ave., Ottawa, ON K1Y 4E9, Canada. email: gjansen@toh.on.ca. 